Hurtado Espinoza, A. K. \& Madueño Serrano, M.L. (2017). Propiedades psicométricas del instrumento prácticas derivadas del aprendizaje social del profesorado. Revista Electrónica Interuniversitaria de Formación del Profesorado, 20 (3), 93-106. DOI: http://dx.doi.org/10.6018/reifop.20.3.291731

\title{
Propiedades psicométricas del instrumento prácticas derivadas del aprendizaje social del profesorado
}

\author{
Ana Karen Hurtado Espinoza, María Luisa Madueño Serrano \\ Instituto Tecnológico de Sonora
}

\section{Resumen}

El objetivo fue establecer las propiedades psicométricas de un instrumento para medir en qué grado las acciones derivadas de la socialización con estudiantes, especialistas educativos-disciplinares y pares académicos contribuyen en la formación docente de los profesores universitarios. Se seleccionó de forma no probabilística a 196 profesores que se encuentran adscritos a una institución pública del Sur de Sonora, de los cuales 100 son mujeres ( $M$ edad=36.5, DE=9.4 años), y 96 hombres ( $M$ edad=42.2, $D E=11.7$ años). Los resultados reflejan que la escala tiene confiabilidad medida por Alfa de Cronbach de .94; en el análisis factorial exploratorio se utilizó el método de ejes principales y rotación Oblimin, se obtuvo una solución de tres factores que explican el $56.46 \%$ de la varianza de los puntajes. En conclusión, el instrumento presenta evidencias de confiabilidad y validez. Además, es una escala potencial para medir las prácticas derivadas del aprendizaje social que contribuyen a la formación docente de los profesores universitarios.

\section{Palabras clave}

Profesores universitarios; formación docente; proceso de aprendizaje.

\section{Psychometric properties derived from social learning practices of university professor}

\section{Abstract}

The aim was to establish the psychometric properties of an instrument to measure the extent to which actions derived from socializing with students, educational-disciplinary specialists and academic peers contribute in teacher education of university professors. It is

Contacto:

Ana Karen Hurtado Espinoza, ana.hurtado@itson.edu.mx, 5 de febrero 818 sur Col. Centro, C.P. 85000. Artículo vinculado al proyecto "La formación docente de los profesores de Educación Superior desde sus prácticas y significados. Una mirada Latinoamericana” financiado por el Programa para el Desarrollo Profesional Docente (PRODEP). 
selected from non-probabilistic way to 196 teachers who are attached to a public institution in Southern Sonora, of which 100 are women ( $M$ age $=36.5, S D=9.4$ years) and 96 men ( $M$ age $=42.2, D E=11.7$ years). The results show that the scale has reliability measured by Cronbach's alpha of .94; in exploratory factor analysis method Oblimin main axes and rotation was used, a solution of three factors that explain $56.46 \%$ of the variance of the scores was obtained. In conclusion, the instrument shows evidence of reliability and validity. Moreover, it is a scale to measure potential social learning practices from contributing to teacher training of university teachers.

\section{Key words}

University professor; teacher training; learning process.

\section{Introducción}

En los últimos veinte años, México ha tenido un crecimiento en matrícula estudiantil y en la apertura de Instituciones de Educación Superior (IES). Esto ha requerido la contratación de profesores universitarios para ejercer la docencia y por ende, para atender el incremento de la matrícula (Rodríguez, 2014; Villa, 2013); sin embargo, se cuestiona el tipo de formación con la que ingresa el profesor que es contratado, debido a que el responsable institucional que contrata le solicita que esté preparado en el área disciplinar en la que se desempeñará, pero no se le exige una preparación previa como docente que le ayude en su actuar frente al grupo de estudiantes (Barreras, Martín \& Cisneros, 2014).

En la actualidad, el país mexicano no cuenta con una política pública que señale que la formación docente del profesor universitario debe ser un requisito para su contratación en las universidades (Heras, 2005; Barrera et al., 2014). Lo anterior conlleva que las autoridades institucionales no consideren este aspecto como algo primordial para el ejercicio de los profesores en las IES, debido a que para ellos la expertis disciplinar del profesor es preponderante para su desempeño como docente; sin embargo, la inexperiencia y el desconocimiento de los docentes en cuestiones de educación, y en particular respecto al desarrollo del proceso de enseñanza-aprendizaje, puede provocar problemas en su práctica docente y por ende en el aprendizaje en los estudiantes (García, 2014; Khan \& Chishti, 2012; Santillán, Hernández \& Cordero, 2013).

Desde los años noventa hasta la actualidad, el Gobierno de México, la Secretaría de Educación Pública (SEP) y algunos organismos educativos han propuesto lineamientos para ejercer programas que contribuyan en la formación de los profesores universitarios, con la finalidad de mejorar la formación en docencia de aquellos grupos de académicos que fueron contratados, pero que no están preparados para la función docente (Brunner, 2007; De Vicenzi, 2009). Aunque estos programas se han llevado a cabo por 25 años, tienen un énfasis en preparar al profesor para la función de investigador, dejando en segundo término la función docente (Barrera et al., 2014), en este sentido, el diseño de los programas y propuestas de preparación en relación con la docencia contradicen la intención original de contribuir a la formación docente del profesorado. En relación a lo anterior, se reflexiona que mejorar la función investigativa y la docente del profesor requieren tener el mismo valor para el Gobierno mexicano y los organismos educativos, debido a que los profesores están formando a los futuros profesionales que velaran por los intereses de la sociedad. De acuerdo con Galaz y Gil (2009) los programas de formación no requieren un crecimiento, sino una modificación sustancial a lo que ya existe. 
La mayoría de las universidades en el país llevan a cabo los programas de formación establecidos por la SEP y el Gobierno, pero se identifica que es fundamental que los responsables institucionales estén convencidos de la importancia de una formación docente para los profesores universitarios, con la intención de que balanceen la preparación de éste actor educativo para la función como investigador y como docente, con el mismo grado de relevancia (Rieg \& Wilson, 2008).

Las IES son autónomas por naturaleza, es decir, cada universidad tiene su normativa, lineamientos de actuación y recurso financiero, lo cual no contribuye a homogenizar las acciones de formación docente que se implementan a nivel nacional, ya que algunas universidades implementan modelos tradicionales para formar a los profesores a partir de cursos, talleres, seminarios (Estévez, 2014; Parra-Moreno, Ecima-Sánchez, Gómez-Becerra \& Almenárez-Moreno, 2010); otras IES llevan a cabo modelos integradores en el que se descentraliza y contextualiza la formación como docente del profesor, estas instituciones capitalizan las experiencias de práctica docente del profesorado como un recurso potencial para su desarrollo (Parra et al., 2010; Rieg \& Wilson, 2008). En este sentido, se han desarrollado investigaciones con el propósito de identificar y comprender aquello que mejore la formación docente de los profesores, enfatizando en acciones que recuperan la experiencia de diferentes actores educativos involucrados en la preparación didácticopedagógica del académico.

Como parte de las acciones que ayudan a mejorar el proceso de formación docente del profesor universitario, Madueño (2013) en un estudio realizado en una universidad pública del Sur de Sonora, identificó por medio de una investigación cualitativa mecanismos de mediación que intervienen en dicho proceso, los cuales se dividen en dos ejes: (1) sujetos que actúan como mediadores, en este eje, entre las principales acciones referidas son: trabajar en colegiado con pares; practicar con profesores mentores para prepararse en el quehacer docente; socializar con expertos disciplinares y docentes; reflexionar sobre la práctica docente a partir de la interacción con el estudiante; (2) objetos que actúan como mediadores, en los cuales se consideran los referentes bibliográficos para buscar y consultar sobre dudas del área docente y disciplinar, participar en dispositivos de formación (ej. cursos, talleres, posgrados, foros y diplomados), y consultar el informe de resultados de la evaluación docente institucional. La autora concluye que estos mecanismos de mediación inciden en la forma de ser y de actuar del profesorado, y que a su vez forman parte de las prácticas que contribuyen a su formación en la función docente.

Madueño, Márquez, Manig y Tapia (2014) puntualizan que el profesor universitario experimenta diversas experiencias de aprendizaje derivadas de su práctica que son cruciales para su formación y para la conformación de su identidad docente; entre las experiencias más significativas son: (a) la socialización con los estudiantes porque a partir del acercamiento con el alumno el profesor cuestiona, reflexiona y cambia su práctica de enseñanza a partir de la búsqueda de alternativas para mejorar su quehacer desde la función docente; (b) la socialización con otros profesores en academia, debido a que en dicho espacio se da la apertura para el intercambio de ideas y estrategias que le permiten al profesor mejorar su quehacer docente. Los autores concluyen que las experiencias antes citadas son una plataforma para el desarrollo y transformación de los profesores, debido a que forman parte del proceso de aprendizaje que cambia la acción humana y el desarrollo de la identidad como docente de este actor educativo, lo anterior, como parte del proceso de socialización que experimentan como ser humano.

La práctica docente del profesor universitario representa un escenario de aprendizaje porque de ella surgen recursos de mediación que contribuyen en la formación docente de este actor educativo (Hurtado, Serna \& Madueño, 2015; Madueño, 2013; Madueño et al., 
2014). En este sentido, se optó por desarrollar un instrumento para medir desde un enfoque cuantitativo en qué grado las prácticas derivadas de la socialización con estudiantes, especialistas educativos-disciplinares y pares académicos contribuyen en la formación docente de los profesores universitarios. Por tal motivo, la investigación parte de la hipótesis de que el instrumento cuenta con evidencias de validez y confiabilidad para medir acciones de socialización con los pares académicos, los especialistas educativosdisciplinares y los estudiantes, variables que permiten utilizarse para la medición del constructo prácticas derivadas del aprendizaje social que contribuyen a la formación docente, del profesor universitario que es contratado por su experiencia disciplinar pero que no cuenta con una acreditación para la docencia.

\section{Perspectiva teórica: aprendizaje social}

El ser humano es concebido como sujeto social y cultural por naturaleza. De acuerdo con esta idea, Wenger (2001) presta atención al individuo en comunidad, así como a su trayectoria en contextos de práctica social. Para dicho autor, las experiencias de aprendizaje que viven las personas dentro del entorno social son un vehículo para el desarrollo de su identidad y de sus procesos de formación o de su transformación. Cabe señalar que el fundamento principal en las aportaciones de origen sociocultural es la concepción social del aprendizaje. Los supuestos de la teoría social enfatizan ideas como las siguientes: las personas son seres sociales por naturaleza; el conocimiento es una cuestión de competencia en relación con empresas valoradas por quien aprende; conocer requiere comprometerse de forma activa con el mundo así como asumir significados (Wenger, 2001).

La postura teórica en la que se fundamenta esta investigación pone en primer plano el principio de que individuo y cultura se co-construyen de manera mutua, por lo que el ser humano, dada su naturaleza social, al participar en una práctica específica es modificado por su contexto, a la vez que él lo modifica al ser parte de dicha cultura (Bruner, 1991; Cole, 2003; Wenger, 2001).

El ser humano aprende cuando participa en acciones situadas o prácticas específicas, las cuales se experimentan al involucrarse en una actividad y a lo largo de una trayectoria en dicha actividad. La postura teórica de la que parte el diseño del instrumento presentado en este documento, sostiene que aprender es un proceso de internalización del conocimiento derivado de la socialización con sujetos que tienen diferentes roles en una misma comunidad de práctica o que guardan relación con ella (Lave \& Wenger, 1991).

El aprendizaje se construye con otros y/o a partir de las aportaciones de otros en y durante el ejercicio de la práctica. Desde esta forma de concebir el aprendizaje, los procesos formativos se construyen o configuran a través de actividades situadas en comunidades de práctica en contextos socioculturales y a su vez, los sujetos aprenden al establecer relaciones con otros sujetos, quienes en su carácter de noveles o expertos favorecen una configuración o reconfiguración de nuevos aprendizajes. En este sentido, se ha identificado que las diferentes prácticas de participación que ayudan al profesorado a aprender a ser y a formarse como docentes guardan una relación directa con procesos de socialización con: los pares docentes, con especialistas en educación y con los propios estudiantes (Hurtado et al., 2015; Madueño, 2013; Madueño, 2014; Madueño et al., 2014).

\section{Metodología}

Se seleccionó de forma no probabilística a 196 profesores universitarios que se encuentran adscritos a una institución pública del Sur de Sonora, en particular de los campus ubicados en los municipios de Cajeme y Navojoa. Se les aplicó el instrumento a 100 mujeres (M 
edad=36.5, $D E=9.4$ años), y 96 hombres ( $M$ edad=42.2, $D E=11.7$ años). La muestra en su totalidad se utilizó para obtener el grado de confiabilidad y para realizar el análisis factorial exploratorio.

El instrumento aplicado midió el constructo prácticas derivadas del aprendizaje social que contribuye a la formación docente, bajo el sustento teórico del aprendizaje social basado en Lave y Wenger (1991). Las variables latentes que se midieron fueron tres:

- Prácticas de participación conjunta entre pares académicos. Se refiere a todas aquellas prácticas relacionadas con el diseño, implementación y evaluación del quehacer como docente, que el profesorado considera que favorecen o contribuyen en su proceso de formación docente.

- Prácticas de participación con especialistas educativos-disciplinares. Son las prácticas que le permite al profesorado adecuar o generar decisiones relacionadas con su quehacer como docente, derivadas de la socialización que tiene con sus exprofesores, capacitadores, expertos disciplinares en la materia y/o en docencia.

- Prácticas de participación con el estudiante. Se refiere a las prácticas realizadas con los alumnos implementadas por el profesor durante el desarrollo de su quehacer docente, las cuales lo conduce a realizar adecuaciones y/o toma de decisiones relacionadas con su quehacer docente.

El instrumento fue una escala tipo Likert con 26 ítems, con cinco opciones de respuesta que fluctúan desde 1 (No contribuye), 2 (Contribuye poco), 3 (Contribuye regular), 4 (Contribuye) y 5 (Contribuye totalmente).

\section{Procedimiento}

Para la recolección de los datos se solicitó autorización a los responsables institucionales, a partir de una carta de acceso al campo de estudio se mostró el objetivo de la investigación y el propósito para la aplicación del instrumento. Asimismo, los profesores dieron su consentimiento informado y voluntario para responder la encuesta, garantizando la confidencialidad de la información que brindaron.

En el análisis de las propiedades psicométricas del instrumento se establecieron evidencias de validez y confiabilidad. Dichos análisis se realizaron con el apoyo del estadístico SPSS (V21). La validez de constructo se realizó a partir del análisis factorial exploratorio. La confiabilidad se obtuvo mediante el Alfa de Cronbach para determinar la consistencia interna de los puntajes obtenidos en la escala.

\section{Resultados}

Para iniciar con los análisis estadísticos fue necesario hacer una prueba de normalidad de los datos, con la finalidad de identificar si estos se encontraban dentro de los parámetros de 3 y -3 en asimetría y curtosis. La escala que midió el constructo prácticas derivadas del aprendizaje social que contribuyen a la formación docente constaba de 26 ítems, sin embargo, se hizo una reducción a 24 reactivos al eliminar dos ítems (identificar las fortalezas y debilidades de los alumnos para implementar estrategias de enseñanza de acuerdo sus necesidades; preguntar al alumno si aprendió durante el desarrollo de las clases) que excedían de los parámetros de normalidad. 


\section{Validez de constructo}

La medida de adecuación muestral de Kaiser-Meyer-Olkin (KMO) y la prueba de esfericidad de Bartlett determinaron la idoneidad de los datos para el análisis factorial exploratorio. Por medio del KMO se obtuvo un valor de .923, de acuerdo con Cea (2004) dicho puntaje es excelente. Por otra parte, la prueba Bartlett resultó significativa $\left(x^{2}=3241.1, p<.000\right)$.

Para el análisis factorial exploratorio se utilizó el método de factores de ejes principales y rotación Oblimin. Se tomó la decisión de eliminar los ítems con un valor de carga factorial superior a .30 en más de dos factores (De Vellis, 2012). A partir de dicho criterio se decidió no incluir en la escala el ítem 14: "generar proyectos educativos o acciones para ayudar aprender a otros con apoyo o asesoría de especialistas en docencia y/o educación" debido a que su carga factorial era superior a .30 en el factor uno y en factor dos.

Tabla 1.

Análisis factorial exploratorio de la escala para medir las prácticas derivadas del aprendizaje social que contribuyen a la formación docente

\begin{tabular}{|c|c|c|c|c|c|c|}
\hline Ítems & & & & s facto & ales & \\
\hline & $M$ & $D E$ & F1 & F2 & $F_{3}$ & $h^{2}$ \\
\hline $\begin{array}{l}\text { 8. Evaluar la pertinencia de los } \\
\text { materiales de apoyo generados } \\
\text { o diseñados para abordar el } \\
\text { desarrollo de las clases. }\end{array}$ & 3.97 & 1.06 & .558 & .235 & .104 & .57 \\
\hline $\begin{array}{l}\text { 4. Dialogar con sus pares sobre } \\
\text { problemas o dificultades } \\
\text { relacionadas con el aprendizaje } \\
\text { de los estudiantes. }\end{array}$ & 4.19 & 1.01 & .569 & .250 & .054 & .55 \\
\hline $\begin{array}{l}\text { 6. Generar casos, problemas o } \\
\text { proyectos en forma conjunta } \\
\text { con profesores (pares) que han } \\
\text { impartido la misma clase. }\end{array}$ & 4.00 & 1.16 & .623 & -.008 & .234 & .61 \\
\hline $\begin{array}{l}\text { 7. Valorar de forma conjunta } \\
\text { casos, problemas o proyectos } \\
\text { abordados en clase. }\end{array}$ & 4.06 & 1.06 & .624 & .056 & .198 & .62 \\
\hline $\begin{array}{l}\text { 5. Generar modificaciones a los } \\
\text { planes de clase. }\end{array}$ & 3.96 & 1.19 & .648 & .035 & .029 & .46 \\
\hline $\begin{array}{l}\text { 9. Someter a validación, de otros } \\
\text { profesores, propuestas propias } \\
\text { en la modalidad de materiales, } \\
\text { actividades o estrategias para el } \\
\text { desarrollo de las clases. }\end{array}$ & 3.56 & 1.29 & .666 & .032 & .138 & .59 \\
\hline $\begin{array}{l}\text { 2. Compartir estrategias de } \\
\text { enseñanza y/o aprendizaje } \\
\text { diseñadas para el desarrollo de } \\
\text { la clase. }\end{array}$ & 4.22 & 1.04 & .785 & -.023 & .022 & .62 \\
\hline $\begin{array}{l}\text { 3. Compartir resultados } \\
\text { derivados de las estrategias de } \\
\text { enseñanza y/o de aprendizaje } \\
\text { empleadas durante las clases. }\end{array}$ & 4.16 & 1.04 & .828 & .088 & -.052 & .69 \\
\hline $\begin{array}{l}\text { 1. Participar en la planeación de } \\
\text { las clases con otros profesores. }\end{array}$ & 3.97 & 1.24 & .921 & -101 & -.108 & .68 \\
\hline 20. Implementar estrategias & 4.44 & .772 & -.044 & .634 & .224 & .54 \\
\hline
\end{tabular}

para que el estudiante salga adelante a pesar de sus problemas personales. 


\begin{tabular}{|c|c|c|c|c|c|c|}
\hline \multicolumn{2}{|l|}{ Ítems } & \multicolumn{5}{|c|}{ Cargas factoriales } \\
\hline $\begin{array}{l}\text { 24. Retroalimentar el trabajo de } \\
\text { clase de los estudiantes. }\end{array}$ & 4.67 & .569 & -.021 & .569 & .198 & .44 \\
\hline $\begin{array}{l}\text { 22. Evaluar las críticas de los } \\
\text { alumnos sobre el desarrollo de } \\
\text { las clases. }\end{array}$ & 4.39 & .849 & .120 & .758 & -.052 & .62 \\
\hline $\begin{array}{l}\text { 21. Solicitar a los alumnos } \\
\text { retroalimentación de cómo } \\
\text { mejorar el proceso de } \\
\text { enseñanza. }\end{array}$ & $4 \cdot 35$ & .861 & .046 & .715 & .034 & .56 \\
\hline $\begin{array}{l}\text { 23. Atender los aspectos de } \\
\text { mejora que le proporcionan sus } \\
\text { estudiantes sobre el proceso de } \\
\text { enseñanza-aprendizaje. }\end{array}$ & 4.44 & .773 & .187 & .844 & -.143 & .75 \\
\hline $\begin{array}{l}\text { 15. Incorporar estrategias } \\
\text { docentes al momento de } \\
\text { impartir las clases, a partir de las } \\
\text { sugerencias de especialistas } \\
\text { docentes. }\end{array}$ & 4.27 & .896 & .100 & .239 & .518 & .52 \\
\hline $\begin{array}{l}\text { 16. Emplear redes sociales para } \\
\text { interactuar con expertos o } \\
\text { especialistas en docencia. }\end{array}$ & 3.66 & 1.22 & -.016 & .100 & .623 & .44 \\
\hline $\begin{array}{l}\text { 18. Emplear recomendaciones } \\
\text { para el ejercicio de la docencia. }\end{array}$ & 4.11 & .919 & -.057 & .263 & .652 & .58 \\
\hline $\begin{array}{l}\text { 17. Identificar recursos } \\
\text { educativos elaborados por } \\
\text { especialistas. }\end{array}$ & 3.99 & 1.04 & .117 & .073 & .637 & .56 \\
\hline $\begin{array}{l}\text { 13. Adoptar estrategias de } \\
\text { enseñanza-aprendizaje } \\
\text { empleadas por profesores que } \\
\text { identifica como modelo a seguir. }\end{array}$ & 4.06 & 1.04 & -.040 & .213 & .661 & .56 \\
\hline $\begin{array}{l}\text { 10. Observar la clase de otros } \\
\text { profesores con experiencia en la } \\
\text { docencia. }\end{array}$ & 3.14 & 1.42 & .113 & -.133 & .644 & .44 \\
\hline $\begin{array}{l}\text { 19. Transferir al propio contexto } \\
\text { de enseñanza aprendizaje lo } \\
\text { aprendido con especialistas en } \\
\text { docencia. }\end{array}$ & 4.14 & 1.01 & .008 & .169 & .693 & .61 \\
\hline $\begin{array}{l}\text { 12. Fungir como profesor } \\
\text { adjunto o como auxiliar durante } \\
\text { el desarrollo de las clases de } \\
\text { otro profesor. }\end{array}$ & 3.15 & 1.44 & .144 & -.169 & .709 & .55 \\
\hline $\begin{array}{l}\text { 11. Recurrir a la asesoría de un } \\
\text { profesor con experiencia } \\
\text { docente. }\end{array}$ & 3.77 & 1.21 & .044 & -.136 & .765 & .55 \\
\hline
\end{tabular}

Se obtuvo una solución de tres factores que explican el $56.46 \%$ de la varianza de los puntajes. La correlación entre los factores 1,2 y 3 muestra que cada factor mide constructos diferentes (ver tabla 2), debido a que las correlaciones no sobrepasan los valores .70 y .80 (De Vellis, 2012). 
Tabla 2.

Correlación entre los factores 1, 2 y 3 derivados del análisis factorial

\begin{tabular}{llll}
\hline \multicolumn{3}{c}{ Correlaciones entre los factores } \\
\hline F1 & & \\
F2 & .49 & - & - \\
F3 & .66 & .50 & \\
\hline
\end{tabular}

Los resultados que se muestran en la Tabla 1 y 2 reiteran que, las variables latentes medidas en el instrumento corresponden a las dimensiones: prácticas de participación conjunta entre pares académicos ( $\mathrm{F} 1)$, prácticas de participación con especialistas educativosdisciplinares (F3), prácticas de participación con el estudiante (F2) y, las cuales surgen desde la teoría que sustenta el diseño de la escala. La solución de los tres factores permite resaltar que los profesores universitarios de este estudio, aprenden conforme se involucran en prácticas situadas derivadas de una actividad de su quehacer docente. En este sentido, la socialización que los profesores tienen con otras personas juega un papel preponderante en su formación docente porque el proceso formativo se fortalece cuando las relaciones que establece con otros configura o reconfigura nuevos aprendizajes en el profesor.

\section{Confiabilidad por dimensiones y reactivos}

La confiabilidad de la escala se presenta en dos modalidades, primero la consistencia interna por cada una de las dimensiones que resultaron del análisis factorial exploratorio, después se presenta la confiabilidad de la escala en su totalidad con la finalidad de identificar si se requiere la reducción del instrumento a partir de las correlaciones de cada reactivo con la escala.

Para establecer la consistencia interna de los puntajes de la dimensión prácticas de participación conjunta entre pares académicos se utilizó el estadístico Alfa de Cronbach, obteniendo un valor de .92, lo cual de acuerdo con De Vellis (2012) se sugiere una reducción de la dimensión planteada, sin embargo, se continuará con todos los ítems porque las correlaciones con la escala fueron superiores al valor de .30 (ver tabla 3 ).

Tabla 3.

Confiabilidad por ítem de la dimensión "prácticas de participación conjunta entre pares académicos"

\begin{tabular}{|c|c|c|c|}
\hline Ítems & $\begin{array}{l}\text { Correlación } \\
\text { del ítem- } \\
\text { escala }\end{array}$ & $\begin{array}{l}\text { Alfa } \\
\text { eliminando } \\
\text { el ítem }\end{array}$ & $\begin{array}{l}\text { Decisión } \\
\text { acerca de } \\
\text { conservar } \\
\text { el ítem }\end{array}$ \\
\hline $\begin{array}{l}\text { 1. Participar en la planeación de las clases } \\
\text { con otros profesores. }\end{array}$ & .74 & .91 & Conservarlo \\
\hline $\begin{array}{l}\text { 2. Compartir estrategias de enseñanza y/o } \\
\text { aprendizaje diseñadas para el desarrollo } \\
\text { de la clase. }\end{array}$ & .73 & .91 & Conservarlo \\
\hline $\begin{array}{l}\text { 3. Compartir resultados derivados de las } \\
\text { estrategias de enseñanza y/o de } \\
\text { aprendizaje empleadas durante las clases. }\end{array}$ & .78 & .91 & Conservarlo \\
\hline $\begin{array}{l}\text { 4. Dialogar con sus pares sobre problemas } \\
\text { o dificultades relacionadas con el } \\
\text { aprendizaje de los estudiantes. }\end{array}$ & .69 & .91 & Conservarlo \\
\hline 5. Generar modificaciones a los planes de & .65 & .92 & Conservarlo \\
\hline
\end{tabular}




\begin{tabular}{|c|c|c|c|}
\hline Ítems & $\begin{array}{l}\text { Correlación } \\
\text { del ítem- } \\
\text { escala }\end{array}$ & $\begin{array}{c}\text { Alfa } \\
\text { eliminando } \\
\text { el ítem }\end{array}$ & $\begin{array}{l}\text { Decisión } \\
\text { acerca de } \\
\text { conservar } \\
\text { el ítem }\end{array}$ \\
\hline $\begin{array}{l}\text { 6. Generar casos, problemas o proyectos } \\
\text { en forma conjunta con profesores (pares) } \\
\text { que han impartido la misma clase. }\end{array}$ & .74 & .91 & Conservarlo \\
\hline $\begin{array}{l}\text { 7. Valorar de forma conjunta casos, } \\
\text { problemas o proyectos abordados en } \\
\text { clase. }\end{array}$ & .75 & .91 & Conservarlo \\
\hline $\begin{array}{l}\text { 8. Evaluar la pertinencia de los materiales } \\
\text { de apoyo generados o diseñados para } \\
\text { abordar el desarrollo de las clases. }\end{array}$ & .70 & .91 & Conservarlo \\
\hline $\begin{array}{l}\text { 9. Someter a validación, de otros } \\
\text { profesores, propuestas propias en la } \\
\text { modalidad de materiales, actividades o } \\
\text { estrategias para el desarrollo de las } \\
\text { clases. }\end{array}$ & .74 & .91 & Conservarlo \\
\hline
\end{tabular}

Nota: elaboración propia.

De igual manera, se determinó la confiabilidad de la dimensión prácticas de participación con el estudiante a partir del Alfa de Croncach obteniendo un valor de .87, de acuerdo con De Vellis dicho puntaje se considera un coeficiente muy bueno (ver tabla 4).

Tabla 4.

Confiabilidad de la dimensión "prácticas de participación con el estudiante"

\begin{tabular}{|c|c|c|c|}
\hline Ítems & $\begin{array}{l}\text { Correlación } \\
\text { del ítem- } \\
\text { escala }\end{array}$ & $\begin{array}{c}\text { Alfa } \\
\text { eliminando } \\
\text { el ítem }\end{array}$ & $\begin{array}{l}\text { Decisión } \\
\text { acerca de } \\
\text { conservar } \\
\text { el ítem }\end{array}$ \\
\hline $\begin{array}{l}\text { 20. Implementar estrategias para que el } \\
\text { estudiante salga adelante a pesar de sus } \\
\text { problemas personales. }\end{array}$ & .63 & .86 & Conservarlo \\
\hline $\begin{array}{l}\text { 21. Solicitar a los alumnos } \\
\text { retroalimentación de cómo mejorar el } \\
\text { proceso de enseñanza. }\end{array}$ & .69 & .85 & Conservarlo \\
\hline $\begin{array}{l}\text { 22. Evaluar las críticas de los alumnos } \\
\text { sobre el desarrollo de las clases. }\end{array}$ & .71 & .84 & Conservarlo \\
\hline $\begin{array}{l}\text { 23. Atender los aspectos de mejora que le } \\
\text { proporcionan sus estudiantes sobre el } \\
\text { proceso de enseñanza-aprendizaje. }\end{array}$ & .71 & .84 & Conservarlo \\
\hline $\begin{array}{l}\text { 24. Retroalimentar el trabajo de clase de } \\
\text { los estudiantes. }\end{array}$ & .78 & .83 & Conservarlo \\
\hline
\end{tabular}

Se estableció la confiabilidad de la dimensión prácticas de participación con especialistas educativos-disciplinares por medio del estadístico Alfa de Cronbach, obteniendo 89 (ver tabla 5), lo cual es considerado una consistencia muy buena para los puntajes de la escala (De Vellis, 2012). 
Tabla 5.

Confiabilidad de la dimensión "prácticas de participación con especialistas educativosdisciplinares"

\begin{tabular}{|c|c|c|c|}
\hline Ítems & $\begin{array}{l}\text { Correlación } \\
\text { del ítem- } \\
\text { escala }\end{array}$ & $\begin{array}{l}\text { Alfa } \\
\text { eliminando } \\
\text { el ítem }\end{array}$ & $\begin{array}{l}\text { Decisión } \\
\text { acerca de } \\
\text { conservar } \\
\text { el ítem }\end{array}$ \\
\hline $\begin{array}{l}\text { 10. Observar la clase de otros profesores } \\
\text { con experiencia en la docencia. }\end{array}$ & .62 & .89 & Conservarlo \\
\hline $\begin{array}{l}\text { 11. Recurrir a la asesoría de un profesor } \\
\text { con experiencia docente. }\end{array}$ & .69 & .88 & Conservarlo \\
\hline $\begin{array}{l}\text { 12. Fungir como profesor adjunto o como } \\
\text { auxiliar durante el desarrollo de las clases } \\
\text { de otro profesor. }\end{array}$ & .67 & .88 & Conservarlo \\
\hline $\begin{array}{l}\text { 13. Adoptar estrategias de enseñanza- } \\
\text { aprendizaje empleadas por profesores } \\
\text { que identifica como modelo a seguir. }\end{array}$ & .69 & .88 & Conservarlo \\
\hline $\begin{array}{l}\text { 15. Incorporar estrategias docentes al } \\
\text { momento de impartir las clases, a partir } \\
\text { de las sugerencias de especialistas } \\
\text { docentes. }\end{array}$ & .64 & .88 & Conservarlo \\
\hline $\begin{array}{l}\text { 16. Emplear redes sociales para } \\
\text { interactuar con expertos o especialistas } \\
\text { en docencia. }\end{array}$ & .63 & .88 & Conservarlo \\
\hline $\begin{array}{l}\text { 17. Identificar recursos educativos } \\
\text { elaborados por especialistas. }\end{array}$ & .69 & .88 & Conservarlo \\
\hline $\begin{array}{l}\text { 18. Emplear recomendaciones para el } \\
\text { ejercicio de la docencia. }\end{array}$ & .69 & .88 & Conservarlo \\
\hline $\begin{array}{l}\text { 19. Transferir al propio contexto de } \\
\text { enseñanza aprendizaje lo aprendido con } \\
\text { especialistas en docencia. }\end{array}$ & .72 & .88 & Conservarlo \\
\hline
\end{tabular}

Por último, para determinar la consistencia interna de los puntajes de la escala en su totalidad, se utilizó el estadístico Alfa de Cronbach, obteniendo .94. De acuerdo con De Vellis (2012) dicho puntaje sugiere una reducción de la escala; sin embargo, se tomó la decisión de conservar todos los ítems, debido a que sus correlaciones fueron superiores a .30 con relación a la escala (ver tabla 6 ).

Tabla 6.

Confiabilidad por ítem de la escala para medir las prácticas derivadas del aprendizaje social que contribuyen a la formación docente

\begin{tabular}{lccc}
\hline Ítems & $\begin{array}{c}\text { Correlación } \\
\text { del ítem- } \\
\text { escala }\end{array}$ & $\begin{array}{c}\text { Alfa } \\
\text { eliminando } \\
\text { el ítem }\end{array}$ & $\begin{array}{c}\text { Decisión } \\
\text { acerca de } \\
\text { conservar } \\
\text { el ítem }\end{array}$ \\
\hline $\begin{array}{l}\text { 1. Participar en la planeación de las clases } \\
\text { con otros profesores. }\end{array}$ & .60 & .94 & Conservarlo \\
2. Compartir estrategias de enseñanza y/o & .65 & .94 & Conservarlo
\end{tabular}

de la clase. 


\begin{tabular}{|c|c|c|c|}
\hline Ítems & $\begin{array}{l}\text { Correlación } \\
\text { del ítem- } \\
\text { escala }\end{array}$ & $\begin{array}{l}\text { Alfa } \\
\text { eliminando } \\
\text { el ítem }\end{array}$ & $\begin{array}{l}\text { Decisión } \\
\text { acerca de } \\
\text { conservar } \\
\text { el ítem }\end{array}$ \\
\hline $\begin{array}{l}\text { 3. Compartir resultados derivados de las } \\
\text { estrategias de enseñanza y/o de } \\
\text { aprendizaje empleadas durante las clases. }\end{array}$ & .69 & .94 & Conservarlo \\
\hline $\begin{array}{l}\text { 4. Dialogar con sus pares sobre problemas } \\
\text { o dificultades relacionadas con el } \\
\text { aprendizaje de los estudiantes. }\end{array}$ & .67 & .94 & Conservarlo \\
\hline $\begin{array}{l}\text { 5. Generar modificaciones a los planes de } \\
\text { clase. }\end{array}$ & .58 & .94 & Conservarlo \\
\hline $\begin{array}{l}\text { 6. Generar casos, problemas o proyectos } \\
\text { en forma conjunta con profesores (pares) } \\
\text { que han impartido la misma clase. }\end{array}$ & .71 & .94 & Conservarlo \\
\hline $\begin{array}{l}\text { 7. Valorar de forma conjunta casos, } \\
\text { problemas o proyectos abordados en } \\
\text { clase. }\end{array}$ & .72 & .94 & Conservarlo \\
\hline $\begin{array}{l}\text { 8. Evaluar la pertinencia de los materiales } \\
\text { de apoyo generados o diseñados para } \\
\text { abordar el desarrollo de las clases. }\end{array}$ & .71 & .94 & Conservarlo \\
\hline $\begin{array}{l}\text { 9. Someter a validación, de otros } \\
\text { profesores, propuestas propias en la } \\
\text { modalidad de materiales, actividades o } \\
\text { estrategias para el desarrollo de las clases. }\end{array}$ & .70 & .94 & Conservarlo \\
\hline $\begin{array}{l}\text { 10. Observar la clase de otros profesores } \\
\text { con experiencia en la docencia. }\end{array}$ & .56 & .94 & Conservarlo \\
\hline $\begin{array}{l}\text { 11. Recurrir a la asesoría de un profesor } \\
\text { con experiencia docente. }\end{array}$ & .60 & .94 & Conservarlo \\
\hline $\begin{array}{l}\text { 12. Fungir como profesor adjunto o como } \\
\text { auxiliar durante el desarrollo de las clases } \\
\text { de otro profesor. }\end{array}$ & .62 & .94 & Conservarlo \\
\hline $\begin{array}{l}\text { 13. Adoptar estrategias de enseñanza- } \\
\text { aprendizaje empleadas por profesores } \\
\text { que identifica como modelo a seguir. }\end{array}$ & .66 & .94 & Conservarlo \\
\hline $\begin{array}{l}\text { 15. Incorporar estrategias docentes al } \\
\text { momento de impartir las clases, a partir } \\
\text { de las sugerencias de especialistas } \\
\text { docentes. }\end{array}$ & .67 & .94 & Conservarlo \\
\hline $\begin{array}{l}\text { 16. Emplear redes sociales para } \\
\text { interactuar con expertos o especialistas } \\
\text { en docencia. }\end{array}$ & .57 & .94 & Conservarlo \\
\hline $\begin{array}{l}\text { 17. Identificar recursos educativos } \\
\text { elaborados por especialistas. }\end{array}$ & .68 & .94 & Conservarlo \\
\hline $\begin{array}{l}\text { 18. Emplear recomendaciones para el } \\
\text { ejercicio de la docencia. }\end{array}$ & .66 & .94 & Conservarlo \\
\hline $\begin{array}{l}\text { 19. Transferir al propio contexto de } \\
\text { enseñanza aprendizaje lo aprendido con } \\
\text { especialistas en docencia. }\end{array}$ & .69 & .94 & Conservarlo \\
\hline $\begin{array}{l}\text { 20. Implementar estrategias para que el } \\
\text { estudiante salga adelante a pesar de sus } \\
\text { problemas personales. }\end{array}$ & .56 & .94 & Conservarlo \\
\hline $\begin{array}{l}\text { S1. Solicitar a los alumnos } \\
\text { retroalimentación de cómo mejorar el } \\
\text { proceso de enseñanza. }\end{array}$ & .52 & .94 & Conservarlo \\
\hline
\end{tabular}




\begin{tabular}{|c|c|c|c|}
\hline Ítems & $\begin{array}{l}\text { Correlación } \\
\text { del ítem- } \\
\text { escala }\end{array}$ & $\begin{array}{c}\text { Alfa } \\
\text { eliminando } \\
\text { el ítem }\end{array}$ & $\begin{array}{l}\text { Decisión } \\
\text { acerca de } \\
\text { conservar } \\
\text { el ítem }\end{array}$ \\
\hline $\begin{array}{l}\text { 22. Evaluar las críticas de los alumnos } \\
\text { sobre el desarrollo de las clases. }\end{array}$ & .52 & .94 & Conservarlo \\
\hline $\begin{array}{l}\text { 23. Atender los aspectos de mejora que le } \\
\text { proporcionan sus estudiantes sobre el } \\
\text { proceso de enseñanza-aprendizaje. }\end{array}$ & .56 & .94 & Conservarlo \\
\hline $\begin{array}{l}\text { 24. Retroalimentar el trabajo de clase de } \\
\text { los estudiantes. }\end{array}$ & .53 & .94 & Conservarlo \\
\hline
\end{tabular}

Nota: elaboración propia.

\section{Discusión}

La escala que mide las prácticas derivadas del aprendizaje social que contribuyen a la formación docente presenta evidencias de validez y confiabilidad, lo cual comprueba la hipótesis planteada, es decir, que el instrumento cuenta con evidencias psicométricas para medir prácticas de participación con los pares académicos, los especialistas educativosdisciplinares y los estudiantes. En este sentido, se apoya la utilización de la escala en investigaciones relacionadas con las prácticas o acciones derivadas de la socialización con estudiantes, especialistas educativos-disciplinares y pares académicos para contribuir en la formación docente de los profesores universitarios.

Los estudios relacionados con los mecanismos de mediación y las experiencias de aprendizaje derivados de la práctica docente fortalecen la escala propuesta (Madueño, 2013; Madueño et al., 2014), debido a que las acciones de socialización con diferentes sujetos configuran y reconfiguran los nuevos aprendizajes de los profesores universitarios, en este caso para contribuir a su proceso formativo en la función docente; es decir, que éste actor educativo construye su aprendizaje docente a partir de actividades situadas en su comunidad de práctica, aprendiendo de los estudiantes, los especialistas educativosdisciplinares y pares académicos (Hurtado et al., 2015; Madueño, 2013; Madueño et al., 2014; Lave \& Wenger, 1991).

\section{Conclusiones}

El objetivo de esta investigación fue reportar las propiedades psicométricas de una escala para medir en qué grado las acciones derivadas de la socialización con estudiantes, especialistas educativos-disciplinares y pares académicos contribuyen en la formación docente de los profesores. Lo anterior, porque se considera que es necesario que los responsables institucionales de dicha formación cuenten con instrumentos que posean evidencias de validez y confiabilidad para el estudio de las diversas prácticas o acciones que mejoran o que influyen en el proceso de formación docente del profesorado en las IES, lo anterior, desde la perspectiva y experiencia vivida de los profesores.

A partir de los resultados obtenidos, se concluye que la escala es un instrumento potencial para medir las prácticas derivadas del aprendizaje social que contribuyen a la formación docente, ya que presenta una validez de constructo aceptable y una confiabilidad que refleja una excelente consistencia interna (De Vellis, 2012). Sin embargo, se sugiere volver aplicar el instrumento a una muestra diferente de profesores, con el propósito de desarrollar un análisis factorial confirmatorio que fortalezca la propiedades reportadas y así obtener un modelo de medida que sustente el constructo. Además, se requiere seguir 
realizando estudios para mejorar y verificar las propiedades psicométricas de esta escala con poblaciones de diferentes contextos socioeconómicos y culturales que existen en México.

Se recomienda que las instituciones recurran con mayor frecuencia a las prácticas situadas que experimentan los profesores durante su quehacer docente, con la finalidad de potencializar su desarrollo formativo (Parra et al., 2010; Rieg \& Wilson, 2008), para ello será relevante la aplicación del instrumento aquí expuesto, ya que permite identificar qué prácticas contribuyen en mayor grado en el proceso formativo del profesorado en la función docente; además este tipo de estudios son relevantes para poder valorar, dinamizar o modificar las estrategias que forman parte del sistema o modelo de formación del profesorado para la mejora de su quehacer docente.

\section{Referencias}

Barrera, M. L., Martín, M. J., \& Cisneros, E. J. (2014). Caracterización del académico de nivel superior: políticas para su formación. En P. Sánchez (Ed.), Docencia y Gestión en la Educación Superior (pp. 1-14). México: Pearson.

Bruner, J. (1991). Actos de significado. Más allá de la revolución cognitiva. Madrid: Alianza.

Brunner, J. J. (2007). Universidad y sociedad en América Latina. México: Universidad Veracruzana. Instituto de Investigación en Educación.

Cea, M. A. (2004). Análisis multivariable. Teoría y práctica en la investigación social. España: Síntesis.

Cole, M. (2003). Psicología cultural. España: Morata.

De Vellis, R. (2012). Scale development. Theory and applications (pp. 104-114). USA: SAGE.

De Vincenzi, A. (2009). Concepciones de Enseñanza y su relación con las prácticas docentes: un estudio con profesores universitarios. Pedagogía Universitaria, 12(2), 87-101.

Estévez, E. H. (2014). Tendencias de la docencia universitaria en México a partir de la perspectiva de los académicos. En H. Muñoz (Ed.), La universidad pública en México. Análisis, reflexiones y perspectivas (pp.165-195). México: MAPorrúa.

Galaz, J. F., \& Gil, M. (2009). La profesión académica en México: Un oficio en proceso de reconfiguración. Revista Electrónica de Investigación Educativa, 11(2), 1-31. Recuperado de http://redie.uabc.mx/redie/article/view/237

García, E. R. (2014). Bajo rendimiento académico universitario. Recuperado de https://docs.google.com/document/d/1SkTAMo5fx3tEiMgb9GfhDqeqamUzdqNHZIf7YvlkTo/edit?pli=1

Heras, L. G. (2005). La política de Educación Superior en México: los programas de estímulos a profesores e investigadores. Educare, 9(29), 207-215.

Hurtado, A. K., Serna, M. L., Madueño, M. L. (2015). Práctica docente del profesor universitario: su contexto de aprendizaje. Profesorado. Revista de currículum y formación del profesorado, 19(2), 216-224.

Khan, S. B., \& Chishti, S. H. (2012). Effects of staff training and development on profesional abilities of university teachers in distance learning systems. The quarterly Review of Distance Education, 13(2), 87-94. 
Lave, J., \& Wenger, E. (1991). Aprendizaje situado. Participación Periférica Legítima. New York: Cambridge University Press.

Madueño, M. L. (noviembre, 2013). Mecanismos de mediación que intervienen en el proceso de formación docente del profesorado universitario en el desarrollo de su práctica. En M. C. Barrón, Ponencia llevada a cabo en el XII Congreso Nacional de Investigación Educativa, Guanajuato, México.

Madueño, M. L. (2014). La Construcción de la Identidad Docente: Un Análisis desde la Práctica del Profesor Universitario. (Tesis doctoral no publicada). Universidad Iberoamericana, Puebla, México.

Madueño, M. L., Márquez, L., Manig, A., \& Tapia, C. S. (2014). El aprendizaje en la práctica del profesor universitario: proceso crucial en la formación de la identidad docente. En S. B. Echeverría, M. T. Fernández, E. Ochoa \& D. Y. Ramos (Ed.), Ambientes de aprendizaje y contexto de desarrollo social (pp. 129-140). México: Pearson Educación.

Parra-Moreno, C., Ecima-Sánchez, I., Gómez-Becerra, M. P., \& Almenárez-Moreno, F. (2010). La formación de los profesores universitarios: una asignatura pendiente de la universidad colombiana. Educación y educadores, 13(3), 421-452.

Rieg, S. A., \& Wilson, B. A. (2008). An Investigation of the Instructional Pedagogy and Assessment Strategies Used by Teacher Educators in two Universities Within a State System of Higher Education. Education, 130(2), 277-294.

Rodríguez, R. (2014). Educación superior y transiciones políticas en México. Revista de la Educación Superior, 43(171), 9-36.

Santillán, V. E., Hernández, E. V., \& Cordero, G. (2013). Formación y procesos socio histórico e institucional: universidades. En P. Ducoing (Ed.), Procesos de formación, volumen 1 2002-2011 (pp. 205-259). México: ANUIES

Villa, L. (2013). Modernización de la Educación Superior, alternancia política y desigualdad en México. Revista de la Educación Superior, 42(167), 81-100.

Wenger, E. (2001). Comunidades de práctica. Aprendizaje, significado e identidad. Barcelona: Paidós. 